IJMMS 29:6 (2002) 313-324

PII. S0161171202011614

http://ijmms.hindawi.com

(c) Hindawi Publishing Corp.

\title{
A MODEL FOR DIP-COATING OF A TWO LIQUID MIXTURE
}

\section{S. B. G. O'BRIEN and M. HAYES}

Received 24 January 2001 and in revised form 19 June 2001

\begin{abstract}
We consider a thin film flow where a flat substrate is coated with a mixture of two miscible liquids, of equal viscosity, and develop a model to predict the evolving coating thicknesses. The developed model can, under certain circumstances, be used as an approximation for the dip-coating of a liquid suspension of a viscous volatile liquid and solid solute as occurs in many industrial applications.
\end{abstract}

2000 Mathematics Subject Classification: 35L40, 76D08, 00A69.

1. Introduction. A thin liquid film consisting of a mixture of two miscible liquids, one volatile (which we will refer to as the solvent), one nonvolatile (referred to as the solute), of equal viscosity, flows under gravity over a flat substrate (see Figure 1.1). We develop a lubrication type model $[11,12,13,15]$ to predict the evolving coating thicknesses [1]. The developed model, can under certain circumstances, be used as an approximation for the dip-coating of a liquid suspension of a viscous volatile liquid and solid solute as occurs in many industrial applications [3, 4, 16]. The coating profile on a flat, finite, long substrate is modelled to find the thickness of coating as a function of distance from the top of the substrate [18]. The emphasis here is placed on obtaining approximate analytic solutions $[5,9]$ rather than numerically solving the governing partial differential equations [17].

We consider the case where the initial mixture thickness is uniform and finite. In [14], we considered a related problem with an infinite initial thickness. After a short time the fluid thickness is thin and the rate of reduction in fluid thickness due to gravity and evaporation are of the same order. Thereafter, when the mixture thickness is very thin there is little flow due to gravity while the evaporation of solvent is the main contributor to the rate of mixture reduction. In the case where the solute and solvent have different viscosities, the model developed here would still provide a reasonable approximation for the process. Initially the viscosity of the solvent would be a good approximation for the viscosity of the mixture. If the viscosity of the solute is the larger (smaller) then as the solvent evaporates the viscosity of the mixture would rise (fall). However, for many practical dip-coating processes, it is known that the slowing down of the flow due to the film becoming very thin dominates the effect of the rise in the viscosity [7].

2. The mathematical model. The basic approach is to use a lubrication approximation to exploit the thinness of the liquid film in simplifying the Navier-Stokes equations. We define the small parameter $\epsilon$ as

$$
\epsilon=\frac{H}{L},
$$




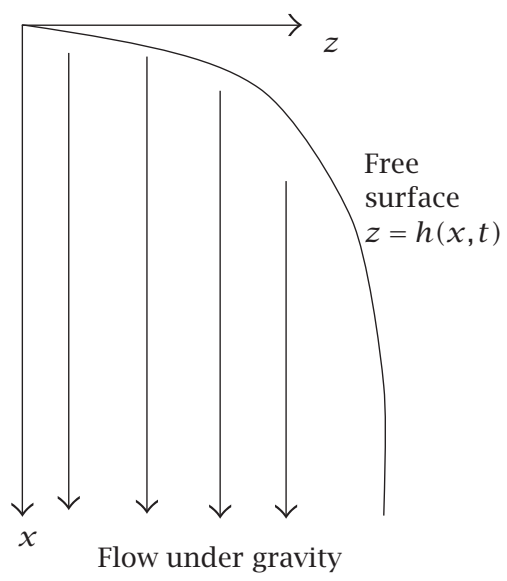

FIGURE 1.1. Schematic of gravity driven flow.

where $L$ is the typical length of the substrate and $H$ is the typical film thickness. If $g$ is the acceleration due to gravity, $\gamma_{s}$ is the surface tension, $\mu, v$ are the dynamic and kinematic viscosities, $h(x, t)$ is the solvent thickness, $s(x, t)$ is the solute thickness, $(u, v)$ is the velocity vector, $\Sigma$ is the mixture thickness $\Sigma=h+s, c(x, t)$ is the ratio of solute thickness to mixture thickness, $s=c(x, t) \Sigma$, [10] we scale

$$
u \sim U \equiv \frac{g H^{2}}{v}, \quad v \sim \epsilon U, \quad x \sim L, \quad z \sim H, \quad p \sim \frac{\gamma_{s} H^{3}}{\mu U L^{3}}, \quad t \sim \frac{L}{U}, \quad s \sim S, \quad \Sigma \sim H,
$$

where $S$ is the initial solute thickness. We assume that the composition of the mixture is uniform initially and due to the thinness of the liquid film the concentration across the film is approximately constant at all times (small Peclet number across the film) and that diffusion in the $x$ direction is dominated by convection (large Peclet number in the $x$ direction). The Peclet number is $\hat{U} \hat{L} / D$ where $\hat{L}$ is an appropriate length scale, $\hat{U}$ is either $U$ or $\epsilon U, v \approx 10^{-6} \mathrm{~m}^{2} \mathrm{~s}^{-1}$ is kinematic viscosity and $D \approx 10^{-9} \mathrm{~m}^{2} \mathrm{~s}^{-1}$ is a typical diffusion constant through a liquid medium. The Peclet number across the film is thus small if the film thickness is less than about $0.1 \mathrm{~mm}$ while along the film the Peclet number is large if the film thickness is larger than about $0.01 \mu \mathrm{m}$.

In the scaled version we thus have

$$
s=\frac{c H}{S} \Sigma, \quad \Sigma=\delta s+h, \delta=\frac{S}{H} \ll 1 .
$$

The governing equations in scaled form become

$$
\begin{gathered}
0=-\frac{\partial p}{\partial x}+\frac{\partial^{2} u}{\partial z^{2}}+1+O\left(\epsilon^{2}\right), \\
0=-\frac{\partial p}{\partial z}+O\left(\epsilon^{2}\right), \\
\frac{\partial u}{\partial x}+\frac{\partial w}{\partial z}=0 .
\end{gathered}
$$


As $H^{2} g / U v=1$ by virtue of the scales (2.2). On $z=0$ there is no-slip while on the free surface $z=\Sigma(x, t)$ we have zero shear stress

$$
\frac{\partial u}{\partial z}=0
$$

The full-dimensional normal stress boundary condition on the free surface $z=\Sigma(x, t)$ is of the form

$$
\mathbf{n} \cdot \mathbf{T} \cdot \mathbf{n}=\gamma_{s} \kappa
$$

where $\mathbf{T}, \mathbf{n}, \kappa$ are the stress tensor, surface unit normal, and surface curvature, respectively [11]. In scaled form, to leading order, this becomes

$$
p=-\frac{\epsilon^{3}}{\mathrm{Ca}\left(\partial^{2} h / \partial x^{2}\right)},
$$

where $\mathrm{Ca}=\mu U / \gamma_{s} \sim \rho g H^{2} / \gamma_{s}$. Using the typical values $\gamma_{s}=7.3 \times 10^{-2} \mathrm{Kg} \mathrm{s}^{-2}, \rho=$ $1.0 \times 10^{3} \mathrm{Kg} \mathrm{m}^{-3}, g=9.81 \mathrm{~m} \mathrm{~s}^{-2}, H \approx 10^{-5} \mathrm{~m}, L \approx 1 \mathrm{~m}$, it is reasonable to choose the distinguished limit $\epsilon^{3} \mathrm{Ca}^{-1} \ll \epsilon^{2}$. From (2.5), we see that the pressure field at leading order is impressed across the film. Hence, in (2.4) the pressure gradient does not contribute at leading order. Thus the velocity field is easily found to be

$$
u(x, z)=-\left[\frac{z^{2}}{2}-h z\right] .
$$

To define the unknown free surface $z=\Sigma(x, t)$, we require an extra free surface condition which we express in terms of mass conservation

$$
\Sigma_{t}=-q_{x} ; \quad q=\int_{z=0}^{z=\Sigma} u(x, z) d z .
$$

We assume that the rate of evaporation of the solvent, $e_{v}$, is constant [10]. With evaporation effects included, the evolution equation for $\Sigma$ takes the form

$$
\Sigma_{t}+\left(\frac{\Sigma^{3}}{3}\right)_{x}+\sigma=0
$$

where $\sigma=e_{v} L / H U$ while the dimensionless solute transport is governed by

$$
s_{t}+\left(\frac{\Sigma^{2}}{3} s\right)_{x}=0
$$

Using the fact that $\Sigma=\delta s+h, \delta \ll 1$ we rewrite these equations as

$$
(\delta s+h)_{t}+\left(\frac{(\delta s+h)^{3}}{3}\right)_{x}+\sigma=0, \quad s_{t}+\left(\frac{(\delta s+h)^{2}}{3} s\right)_{x}=0
$$

which reduces to

$$
\begin{gathered}
h_{t}+\left(\frac{h^{3}}{3}\right)_{x}+\sigma+O(\delta)=0, \\
s_{t}+\left(\frac{h^{2}}{3} s\right)_{x}+O(\delta)=0 .
\end{gathered}
$$


The boundary conditions in dimensional variables (using an asterisk to denote this) are

$$
\Sigma^{*}\left(x^{*}, 0\right)=f^{*}\left(x^{*}\right), \quad h^{*}\left(x^{*}, 0\right)=(1-\delta) f^{*}, \quad s^{*}\left(x^{*}, 0\right)=\delta f^{*}
$$

which in scaled form become

$$
\Sigma(x, 0)=\frac{f^{*}}{H}, \quad h(x, 0)=\frac{(1-\delta) f^{*}}{H}, \quad s(x, 0)=\frac{f^{*}}{H}
$$

which to leading order in $\delta$ are $h(x, 0)=f^{*} / H, s(x, 0)=f^{*} / H$. We consider the case where the initial film is initially uniform and finite, that is,

$$
h(x, 0)=h_{0}, \quad x \geq 0,
$$

or $h(x, 0)=h_{0} \hat{H}(x)$ where $\hat{H}$ is the Heaviside step function.

2.1. The solvent problem. We consider (2.15) subject to the initial condition (2.19).

2.1.1. A shock solution. There is a theoretical shock solution where the initial condition is propagated downwards with shock speed $c=\left(q_{-}-q_{+}\right) /\left(h_{-}-h_{+}\right)=(1 / 3) h_{0}^{2}$ if we recall that $q=h^{3} / 3$. The solution is $h(x, t)=0, x<(1 / 3) h_{0}^{2} t, h(x, t)=h_{0}-\sigma t$, $x>(1 / 3) h_{0}^{2} t$ so the shock is located at $x_{s}=(1 / 3) h_{0}^{2} t$ and it is easy to see that this is a solution of the problem. In the limiting case of slow evaporation where $\sigma=0$, we can easily show that this solution is unstable. We first use a coordinate transformation to fix the position of the shock by setting $z=x-(1 / 3) h_{0}^{2} t, \tau=t, v(z, \tau)=h(x, t)$ in which case the governing equation becomes $v_{\tau}+v_{z}\left(v^{2}-(1 / 3) h_{0}^{2}\right)=0$ and we wish to investigate the stability of the stationary shock $v=0, z<0 ; v=h_{0}, z>0$. For $z<0$ we set $v=0+\delta_{1} \exp (\omega t) f(z)$ with $\delta_{1} \ll 1$ and to leading order in $\delta$ we quickly obtain: $d f / d z=(1 / 3) h_{0}^{2} \omega f$ and so $f=A \exp \left((1 / 3) h_{0}^{2} \omega z\right)$ for some arbitrary $A$. As we also require that $f \rightarrow 0$ as $z \rightarrow-\infty$ we must have $\omega>0$ and the solution is unstable. Similarly for $z>0$ we find that the solution is unstable. In each of these situations the perturbed solution on the real line is defined in a piecewise fashion and is supplemented by a condition on the shock of the form $C(\tau)=\left(q_{-}-q_{+}\right) /\left(h_{-}-h_{+}\right)=B \delta_{1} \exp (\omega \tau)$ where $B$ is defined by the values of the film thickness and the flux on either side of the shock.

This form of shock instability is well known and we could have predicted it by interpreting (2.15) as a kinematic wave equation. The kinematic wave speed is $\partial\left(h^{3} / 3\right) / \partial h=$ $h^{2}$. When $x>(1 / 3) h_{0}^{2} t$, we have $h=h_{0}>0$ so small disturbances propagate away from the shock and in this way the shock will tend to break up.

In the case where $\sigma>0$, the basic shock solution is unsteady due to the evaporation effects even though the location of the shock has been fixed via coordinate transformation. In this instance we have a static but time-dependent shock [2] $h=0$, $z<0 ; h=h_{0}-\sigma \tau, z>0$. For $z<0$ we set $v=\delta f(\tau, z)$ with $\delta \ll 1$ and disturbances evolve according to $f_{\tau}-(1 / 3) h_{0}^{2} f_{z}=0$ with the general solution $f=F\left(z+(1 / 3) h_{0}^{2} \tau\right)$ where $F$ describes the form of the disturbance. This describes a wave propagating in the $-z$ direction so the disturbance is not captured by the shock and the basic solution is unstable. Similarly, when $z>0$ we set $v=h_{0}-\sigma \tau+\delta f(\tau, z)$ and we find that 
$f=F(z-D(\tau))$ where $d(\tau)=\left(h_{0}-\sigma \tau\right)^{2}-(1 / 3) h_{0}^{2}$ and $d D(\tau) / d \tau=d(\tau)$. Signals thus travel on the space time curves $x-D(\tau)=$ constant. For small times $\tau$ we note that $d(\tau)>0$ and thus $D(\tau)>0$ and so the disturbance $F$ propagates with positive velocity away from the shock with consequent instability.

2.1.2. A continuous solution. As the shock solution is unstable, we seek an alternative solution by assuming the existence of a fan type solution emanating from $x=0$. Thus at any time $t>0$ far enough down the film at $x=x_{f}$ the disturbance caused by the non-uniformity at $x=0$ will not yet have any influence and the film thickness will be uniform, though less than its initial value due to evaporation, that is, $h_{0}-\sigma t$. For $x<x_{f}$, we expect a monotonically increasing profile arising from the expansion fan emanating from $x=0$. At the top of the film there will be an interval $x<x_{d}$ where the liquid thickness is zero as a result of evaporation and the fact that the film is not being replenished from above.

By demanding continuity of the film thickness, $h(x, t)$ on the whole domain can be written as a piecewise smooth function

$$
h(x, t)= \begin{cases}0, & 0 \leq x<x_{d} \\ \left(-\frac{\sigma t}{2}+\frac{1}{t} \sqrt{t x-\frac{\sigma^{2} t^{4}}{12}}\right), & x_{d} \leq x<x_{f} \\ h_{0}-\sigma t, & x_{f} \leq x .\end{cases}
$$

The drying line (i.e., the line in $x t$ space corresponding to $h=0$ ) is given by

$$
x_{d}=\frac{1}{3} \sigma^{2} t^{3},
$$

and as $h(x, t)$ is continuous at $x_{f}$ we have the following relationship between $x_{f}$ and $x_{d}$ :

$$
x_{f}=x_{d}+h_{0} t\left(h_{0}-\sigma t\right) .
$$

2.2. The solute problem. From (2.16) the equation describing the solute thickness is

$$
\frac{\partial s}{\partial t}+\frac{1}{3} h^{2} \frac{\partial s}{\partial x}+\frac{2}{3} h s \frac{\partial h}{\partial x}=0
$$

The initial condition for the solute is

$$
s(x, 0)=h_{0} \quad \text { or } \quad s(x, 0)=h_{0} \hat{H}(x) .
$$

The characteristics are defined by

$$
\frac{d x}{d t}=\frac{1}{3} h^{2}
$$

where $h$ is defined in (2.20) and we would like to obtain an explicit (as distinct from parametric) representation if possible. In Figure 2.1, we use (2.21) to plot the drying line and via (2.22) the disturbance line. The disturbance line is the curve that defines 


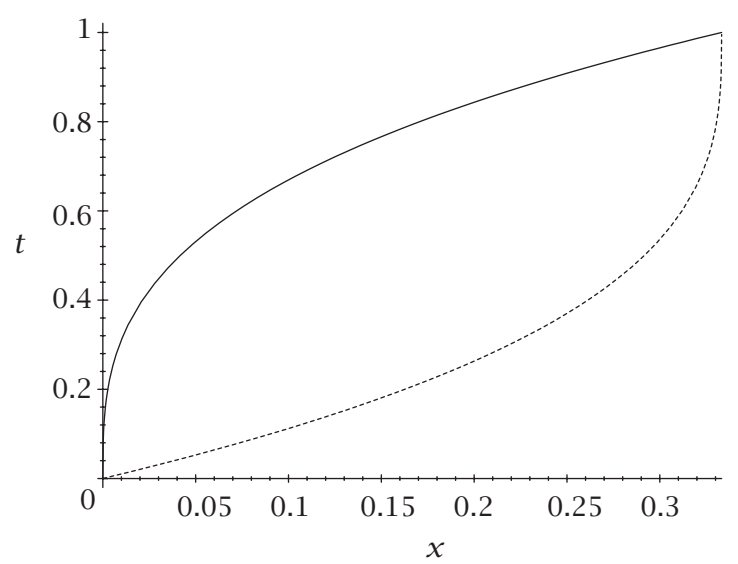

FIGURE 2.1. The drying line (solid) above the disturbance line (dashed) for $h_{0}=1$.

when and where the fluid thickness changes to the flat profile $h_{0}-\sigma t$. Substituting (2.20) into (2.25), we obtain

$$
\frac{d x}{d t}=l(t)+\frac{x}{3 t}+m(t) \sqrt{x+n(t)},
$$

where

$$
l(t)=\frac{\sigma^{2}}{18} t^{2}, \quad m(t)=-\frac{\sigma}{3} \sqrt{t}, \quad n(t)=-\frac{\sigma^{2}}{12} t^{3} .
$$

Writing $\alpha(t)=x(t)+n(t)$, (2.26) becomes

$$
\frac{d \alpha(t)}{d t}=f(t) \sqrt{\alpha(t)}+g(t) \alpha(t)+q(t),
$$

where

$$
f(t)=-\frac{\sigma}{3} \sqrt{t}, \quad g(t)=\frac{1}{3 t}, \quad q(t)=-\frac{\sigma^{2}}{6} t^{2} .
$$

From [6], the solution of (2.28) is

$$
\alpha(t)=\left(\frac{q(t)}{f(t)}\right)^{2} x(t)
$$

where $\chi(t)$ satisfies

$$
\Upsilon=-\int \frac{d \chi}{\sqrt{X}-\gamma \chi+1}+\int \frac{(f(t))^{2}}{q(t)} d t
$$

$\mathrm{Y}$ is an arbitrary constant labeling the characteristics and the constant $\gamma$ satisfies

$$
\frac{d z(t)}{d t}-g(t) z(t)=\gamma q(t), \quad z(t)=\left(\frac{q(t)}{f(t)}\right)^{2}
$$


and we easily find that $\gamma=-4$ and

$$
\Upsilon=-\int \frac{d \chi}{\sqrt{X}+4 x+1}-\frac{2}{3} \int \frac{1}{t} d t
$$

We now define

$$
a(x)=\frac{1}{4} \log (4 x+\sqrt{x}+1)-\frac{1}{2 \sqrt{15}} \tan ^{-1}\left(\frac{8 \sqrt{x}+1}{\sqrt{15}}\right) .
$$

The equations for the characteristics in the region between the drying line and the disturbance line are obtained from (2.33) giving

$$
\xi_{2}=-a(x)-\frac{2}{3} \log t
$$

where $\xi_{2}$ defines the particular characteristic with $a(\chi)$ defined by (2.34) and

$$
x(t)=\frac{x(t)-\left(\sigma^{2} / 12\right) t^{3}}{\left(\sigma^{2} / 4\right) t^{3}}
$$

The characteristics in the region below the disturbance line are obtained by substituting (2.20) into (2.25) to obtain

$$
\frac{d x}{d t}=\frac{1}{3}\left(h_{0}-\sigma t\right)^{2}
$$

giving

$$
\xi_{1}=x-\frac{1}{3}\left(h_{0}^{2} t-h_{0} \sigma t^{2}+\frac{1}{3} \sigma^{2} t^{3}\right),
$$

where $\xi_{1}$ defines the characteristic. We define $t_{0}$ and $t_{1}$ to be the unique times a characteristic intersects the drying and disturbance lines, respectively. The characteristics below the disturbance line given by (2.38) intersect the $x$ axis at $\xi_{1}$. From the disturbance line (2.22) and the equation of the characteristic below the disturbance line (2.38), the $x$ coordinate of their point of intersection is

$$
x_{f}=\frac{3}{2} \xi_{1} .
$$

Using (2.39) and (2.38), the other coordinate of intersection $t_{1}$ between the disturbance line and the characteristic below the disturbance line which lies between 0 and $\left(9 \xi_{1} / 2 \sigma^{2}\right)^{1 / 3}$ is obtained numerically as a function of $\xi_{1}$. The value of $t$ along the characteristic below the disturbance which lies between 0 and $t_{1}\left(\xi_{1}\right)$ is numerically expressed as a function of $x$ and $\xi_{1}$, using (2.38). $\xi_{2}$ is expressed as a function of $\xi_{1}$ by substituting the coordinates of the intersection point between the disturbance line and the characteristic below the disturbance line into the equation for the characteristic above the disturbance line (2.35). The equation of the drying line (2.21) and the equation of the characteristic above the disturbance line (2.35) are solved for $t_{0}$ as a function of $\xi_{2}\left(\xi_{1}\right)$. The value of $t$ along the characteristic above the disturbance line which lies between $t_{1}\left(\xi_{1}\right)$ and $t_{0}\left(\xi_{1}\right)$ is numerically expressed as a function of $x$ and $\xi_{1}$ using (2.35). A complete characteristic is defined as a function of $x$ and $\xi_{1}$ if we choose the value of $t$ along the characteristic below or above the disturbance 


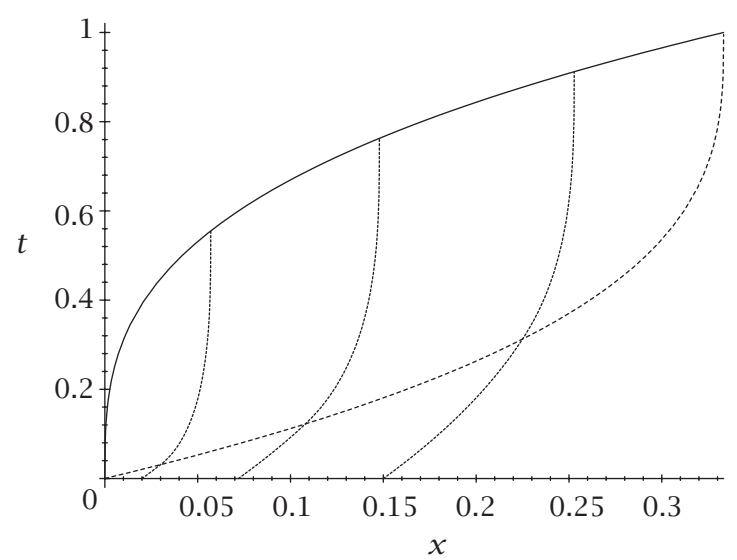

FIGURE 2.2. The drying line (solid) above the disturbance line (dashed) and related characteristics (dotted) for $h_{0}=1$.

line if $x<(3 / 2) \xi_{1}$ or $x \geq(3 / 2) \xi_{1}$, respectively. Finally, we plot in Figure 2.2 some characteristics starting at $x=\xi_{1}$ and ending at $x=(1 / 3) \sigma^{2} t_{0}\left(\xi_{1}\right)^{3}$ on the drying line.

We now make the following change of independent variables:

$$
\eta=t, \quad \xi= \begin{cases}x-\frac{1}{3}\left(h_{0}^{2} t-h_{0} \sigma t^{2}+\frac{1}{3} \sigma^{2} t^{3}\right), & (x, t) \text { is below the disturbance line, } \\ -a(x)-\frac{2}{3} \log t, & (x, t) \text { is between the disturbance } \\ x, & \text { and the drying lines, } \\ & (x, t) \text { is above the drying line. }\end{cases}
$$

Hence (2.23) becomes

$$
\frac{d s}{d \eta}=-\frac{2}{3} h s \frac{\partial h}{\partial x}
$$

where $s=s(\xi, \eta)$ and the initial condition becomes

$$
s(\xi, 0)=h_{0}
$$

From (2.20), it can be seen that $\partial h / \partial x=0$ when $x<x_{d}$ and $x>x_{f}$. Hence the righthand side of (2.41) is nonzero when in the middle region, that is, when $x_{d} \leq x \leq x_{f}$. So (2.41) becomes

$$
\frac{d s}{d \eta}= \begin{cases}0, & (x, t) \text { is below the disturbance line, } \\
s\left[\frac{1}{\sqrt{\left(36 / \sigma^{2}\right) x(\xi, \eta) / \eta-3 \eta^{2}}}-\frac{1}{3 \eta}\right], & \begin{array}{l}
(x, t) \text { is between the disturbance } \\
\text { and the drying lines, } \\
0,
\end{array} \\
(x, t) \text { is above the drying line. }\end{cases}
$$


As $t_{0}$ and $t_{1}$ are the times a characteristic intersects the drying and disturbance lines, respectively, using the initial condition (2.42), (2.43) becomes

$$
\int_{h_{0}}^{s} \frac{d s}{s}=\int_{t_{1}}^{t_{0}}\left[\frac{1}{\sqrt{\left(36 / \sigma^{2}\right) x(\xi, \eta) / \eta-3 \eta^{2}}}-\frac{1}{3 \eta}\right] d \eta
$$

which reduces (with $\psi=\eta / t_{0}$ ) to

$$
s(\xi)=c_{0} h_{0} \sqrt[3]{\frac{t_{1}}{t_{0}}} \exp \left[\int_{t_{1} / t_{0}}^{1} \frac{d \psi}{\sqrt{\left(36 / \sigma^{2}\right)\left(x(\xi, \eta) / t_{0}^{3}\right) 1 / \psi-3 \psi^{2}}}\right]
$$

and it follows that

$$
-a(1)=-a\left(\frac{x(\eta) / t_{0}^{3}-\left(\sigma^{2} / 12\right) \psi^{3}}{\left(\sigma^{2} / 4\right) \psi^{3}}\right)-\frac{2}{3} \log \psi .
$$

To evaluate $s(\xi)$ in (2.45), we first express $t_{0}$ and $t_{1}$ as functions of $\xi$. Substituting (2.36) and (2.21) into (2.40) we have

$$
t_{0}=\exp \left(-\frac{3}{2}(\xi+a(1))\right)
$$

To obtain $t_{1}$ as a function of $\xi$, we solve for the intersection point of the equation of the characteristic (2.40) and the equation of the disturbance line (2.22). From (2.46) we solve for $x / t_{0}^{3}$ as a function of $\psi$ so as to enable us to compute the integral in (2.45) where $x / t_{0}^{3}$ lies between 0 and $\sigma^{2} / 3$. We can now integrate (2.45) and obtain $s$ as a function of $\xi$. Substituting the equation for the drying line (2.21) into (2.47), we obtain

$$
\xi(x)=-a(1)-\frac{2}{9} \log \left(\frac{3}{\sigma^{2}} x\right) .
$$

Substituting (2.48) into the numerical solution for $s(\xi)$, we obtain $s$ expressed as a function of $x$. In Figure 2.3, we plot the solute thickness as a function of the distance from the top of the substrate for a number of finite initial fluid thicknesses together and compare this with the solute profile corresponding to an infinite initial fluid thickness (smooth curve). In Figure 2.4 we demonstrate the effect of varying the evaporation parameter $\sigma$.

2.2.1. The solute profile before $h=0$. From the drying line (2.21) and the fluid profile thickness (2.20), it can be seen that the process time for the substrate to be fully dry is the minimum of $\sqrt[3]{3 L / \sigma^{2}}$ and $h_{0} / \sigma$ where $L$ is the substrate length. We will now find the scaled solute thickness as a function of $x$ at some time $\bar{t}$ less than the process time, if all the solvent $h$ were instantaneously to evaporate at time $\bar{t}$.

The solution of the scaled solute profile at a time before the substrate is fully dry with a finite initial fluid thickness is a piecewise smooth function with the domain broken into three parts. It is clear that all solvent has evaporated from $x=0$ to the $x$ coordinate of the point of intersection of the drying line and the line $t=\bar{t}$. Consequently, the solution of the scaled solute profile in this domain is given by (2.45) 


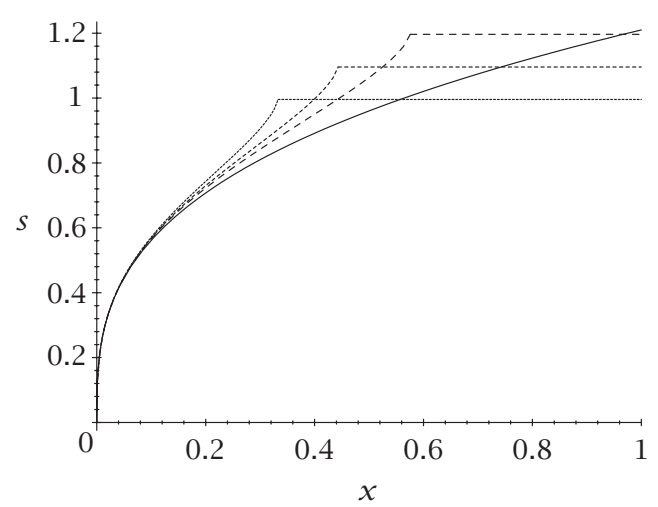

FIGURE 2.3. Solute thickness for $h_{0}=1,1.1,1.2$ and the solute profile for an infinite initial fluid thickness (solid). $\sigma=c_{0}=1$.

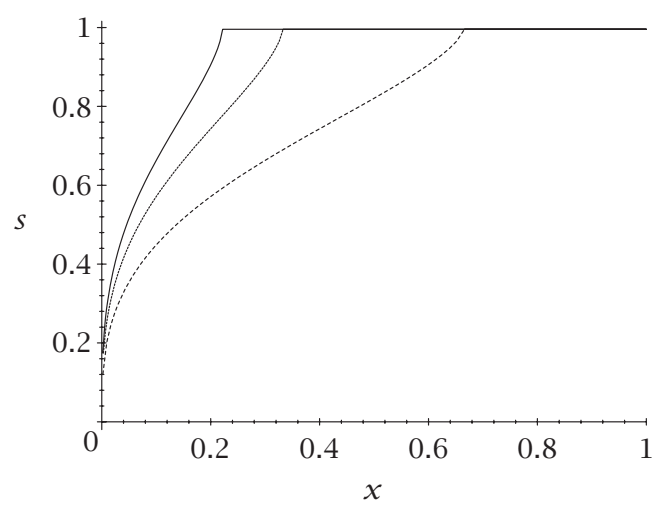

FIGURE 2.4. Solute thickness for $h_{0}=c_{0}=1, \sigma=1.5$ (solid), $\sigma=1$ (dotted), $\sigma=0.5$ (dashed).

using (2.46), (2.47), and (2.48). From this point to the end of the substrate the profile can be obtained from (2.20).

In the second region, the development is similar to that given in Section 2.2 with $t_{0}$ replaced by $\bar{t}$. In Figure 3.1, we plot $s(x)$ against $x$ at $t=\bar{t}$. In Figure 3.2, we superimpose plots of $s(x)$ at $t=\bar{t}$ and when $h=0$, against $x$ for a finite initial fluid thickness.

3. Discussion. We have developed a simplified model for the flow under gravity of a two liquid mixture with one volatile component. We assume that evaporation of the solvent takes place at a constant rate [10]. In the thin film approach used here, the problems for the evolution of the nonvolatile component (solute) and volatile component (solvent) partially uncouple and it becomes possible to obtain approximate solutions without numerically integrating the governing partial differential equations. Solutions for the solvent can be found explicitly; numerical quadrature is required to resolve the solute problem. 


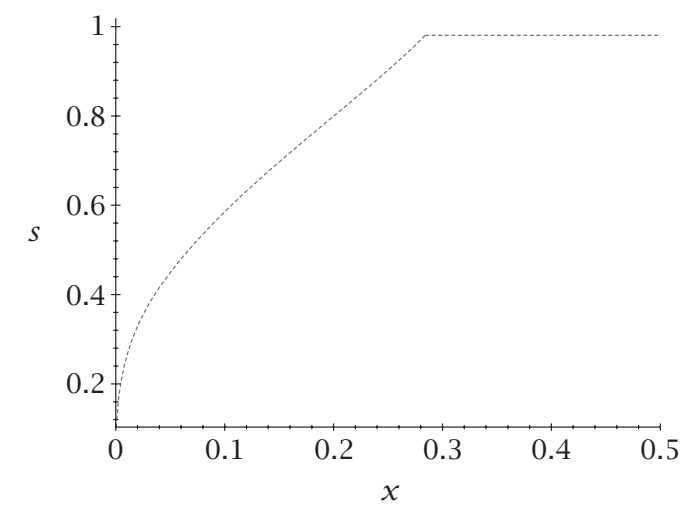

FIGURE 3.1. Solute thickness at $\bar{t}=1 / 2$ with $\sigma=c_{0}=h_{0}=1$.

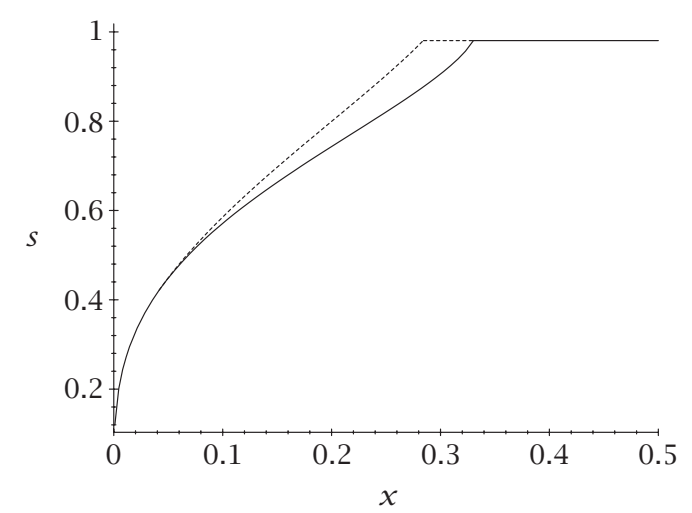

FIGURE 3.2. Solute thickness at $\bar{t}$ (dashed), and when all the solvent has evaporated for $\sigma=c_{0}=h_{0}=1, \bar{t}=1 / 2$.

The solutions are split into three regions, an uppermost region where all the solvent has evaporated, an intermediate region where evaporation and gravity effects thin the film and a lower region where the disturbance created by the initial condition has not yet propagated and where the mixture thins purely as a result of evaporation. This model can also be used to approximate the classical dip-coating process where the solute is in the solid phase. One complication is that as the solvent level decreases, the viscosity can increase significantly, an effect not incorporated in the present model. A perturbation model for the case where the rise in viscosity is small could easily be developed along the lines of the present paper. Moreover, it is well known that in most practical situations [8], the film has already become so thin that the flow is negligible before the viscosity changes significantly so the assumption of constant viscosity is reasonable.

ACKNOWLEDGEMENT. We acknowledge the assistance of D. Tocher in the preparation of the figures. 


\section{REFERENCES}

[1] B. V. Deryagin and S. M. Levi, Film Coating Theory, Focal Press, London, 1964.

[2] P. G. Drazin and W. H. Reid, Hydrodynamic Stability, Cambridge Monographs on Mechanics and Applied Mathematics, Cambridge University Press, Cambridge, 1981.

[3] A. G. Emslie, F. T. Bonner, and L. G. Peck, Flow of a viscous liquid in a rotating disk, J. Appl. Phys. 29 (1958), 858-862.

[4] P. Groenveld, Drainage and withdrawal of liquid films, AIChE J. 17 (1971), 489-490.

[5] H. Jeffreys, Draining of a vertical plate, Proc. Cambridge Philos. Soc. 26 (1930), 204-205.

[6] E. Kamke, Differentialgleichungen. Lösungsmethoden und Lösungen. Teil I: Gewöhnliche Differentialgleichungen. 6. Aufl.; Teil II: Partielle Differentialgleichungen erster Ordnung für eine gesuchte Funktion. 4. Aufl, Akademische Verlagsgesellschaft Geest \& Portig K.-G., Leipzig, 1959 (German).

[7] J. A. Lammers and S. B. G. O'Brien, Effects of evaporation during spin-coating, The Mechanics of Thin Film Coatings (P. H. Gaskell and M. D. Savage, eds.), World Scientific Press, 1996, pp. 397-403.

[8] J. H. Lammers, private communication.

[9] J. H. Lammers, M. Decre, and S. B. G. O’Brien, Spin-coating over topography, Second European Coating Symposium (Strasbourg), 1997.

[10] D. Meyerhofer, Characteristics of resist films produced by spinning, J. Appl. Phys. (1978), 3993-3997.

[11] S. B. G. O'Brien, On Marangoni drying: Nonlinear kinematic waves in a thin film, J. Fluid Mech. 254 (1993), 649-670.

[12] _ Linear stability of rimming flows, to appear in Quart. Appl. Math., 2002.

[13] S. B. G. O'Brien and E. G. Gath, The location of a shock in rimming flow, Phys. Fluids 10 (1998), 1-3.

[14] S. B. G. O'Brien and M. Hayes, Approximate models for dip-coating of suspensions, in preparation.

[15] S. B. G. O'Brien and L. W. Schwartz, Theory and modelling of thin liquid films, to appear in Encyclopedia of Surface and Colloid Science, 2002.

[16] L. D. Rosenberg and R. L. Beiter, Impact of new technology and changing Practices in the automotive coating business, AIChE Symposium Series on New Polymer Technology for Auto Body Exteriors, 1989.

[17] L. E. Scriven, Physics and application of dip-coating and spin-coating, Better Ceramics Through Chemistry III (C. J. Brinker, D. E. Clark, and D. R. Ulrich, eds.), Materials Research Society, Pittsburgh, 1988, pp. 717-729.

[18] J. Van Rossum, Viscous lifting and drainage of liquid, Appl. Sci. Res. 7 (1958), 121-144.

S. B. G. O'Brien: Department of MATHEMAtics, UniVersity of Limerick, Limerick, IRELAND

E-mail address: stephen.obrien@u 1.ie

M. HAYES: DEPARTMENT OF MATHEMATICS, UNIVERSITY OF LIMERICK, LIMERICK, IRELAND 


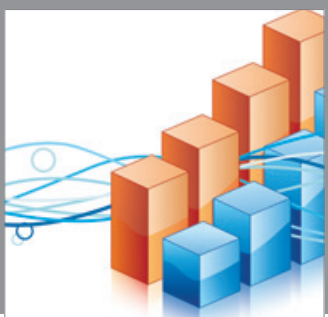

Advances in

Operations Research

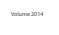

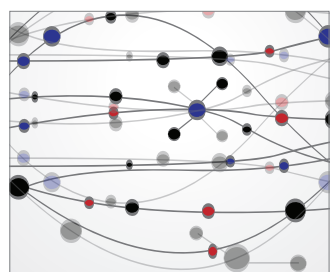

\section{The Scientific} World Journal
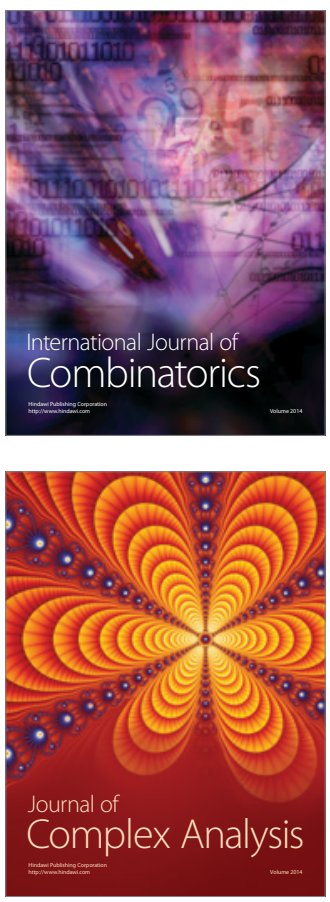

International Journal of

Mathematics and

Mathematical

Sciences
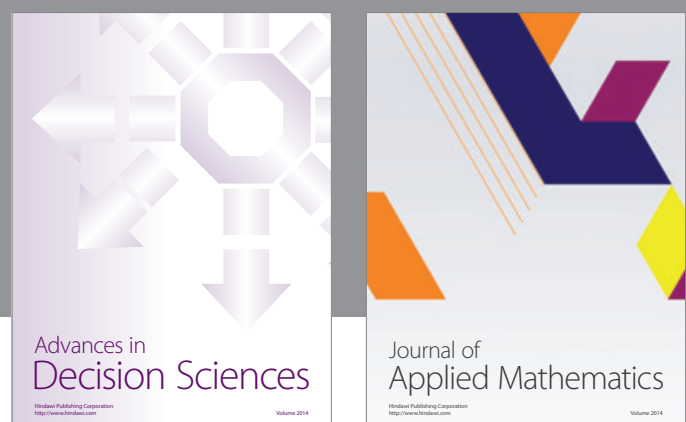

Journal of

Applied Mathematics
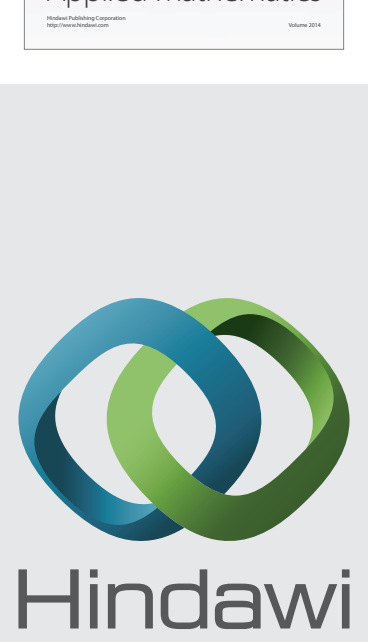

Submit your manuscripts at http://www.hindawi.com
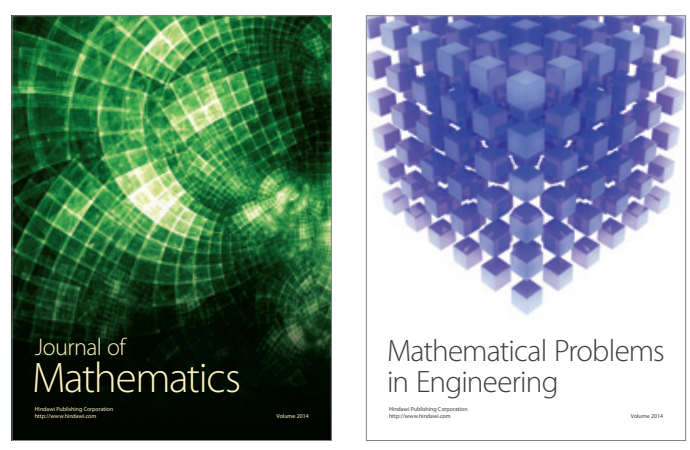

Mathematical Problems in Engineering
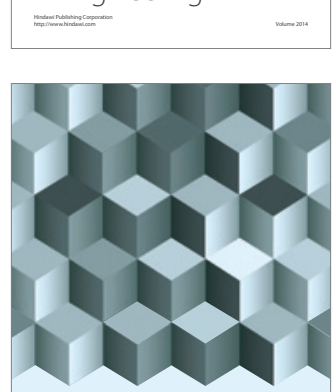

Journal of

Function Spaces
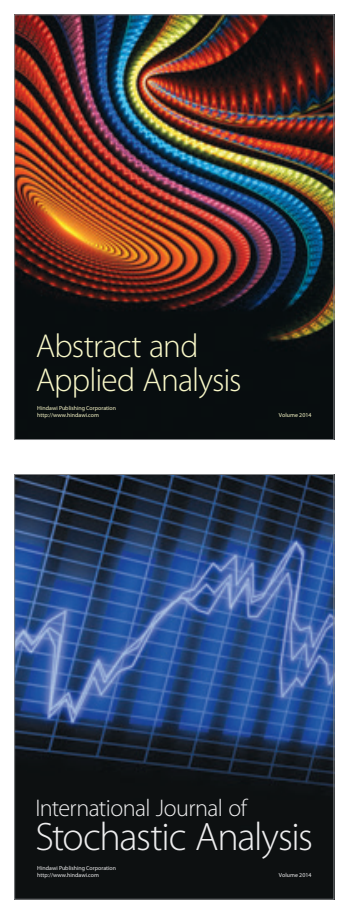

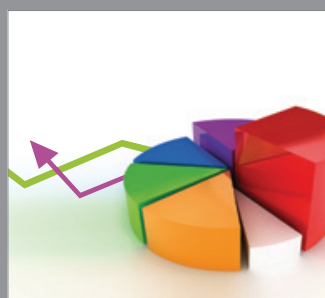

ournal of

Probability and Statistics

Promensencen
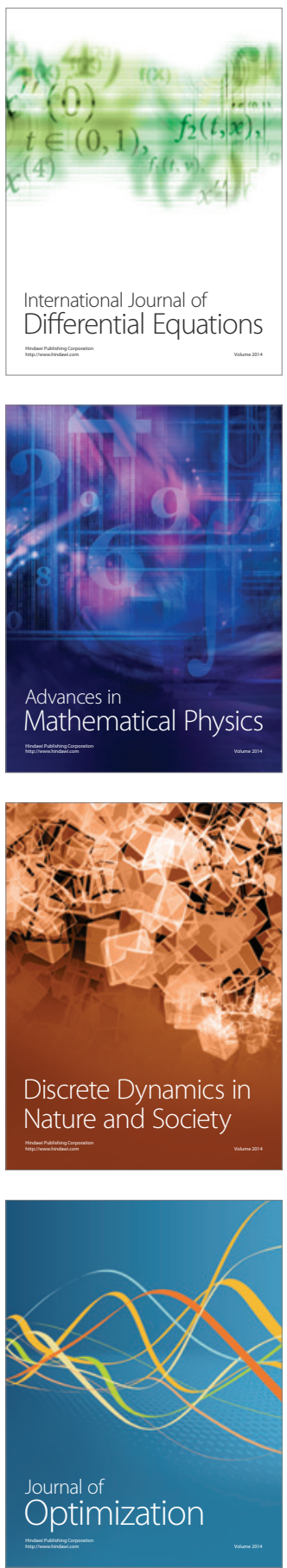